\title{
Clinical Study of Bell's Palsy at Tertiary Care: Our Experience
}

\author{
M. B. Bharathi ${ }^{1} \quad$ Thanzeem Unisa ${ }^{1} \quad$ Swathi Chandresh ${ }^{2}$ Venkatesh C.R. ${ }^{3} \quad$ Harsha S. $^{4}$
}

\author{
${ }^{1}$ Department of Otorhinolaryngology, JSS Academy of Higher \\ Education and Research (JSSAHER), Mysuru, Karnataka, India \\ ${ }^{2}$ Department of Otorhinolaryngology, All India Institute of Speech \\ and Hearing, Mysuru, Karnataka, India \\ ${ }^{3}$ Department of Medicine, JSS Academy of Higher Education and \\ Research (JSSAHER), Mysuru, Karnataka, India \\ ${ }^{4}$ Department of Neurology, JSS Academy of Higher Education and \\ Research (JSSAHER), Mysuru, Karnataka, India
}

Address for correspondence Thanzeem Unisa, MBBS, MS, \#14/A 6th Cross, Shivaji Road, N. R. Mohalla, Mysuru 570007, Karnataka, India (e-mail: thanzeem@gmail.com).

Ann Otol Neurotol ISO 2019;2:16-20

\begin{abstract}
Keywords

- Bell's palsy

- topodiagnostic tests

- House-Brackmann facial nerve grading

- electrodiagnostic test

- electroneuromyography

Introduction Bell's palsy (BP) is the common cause of facial palsy. This study aims to report and analyze the age, sex distribution, symptomatology, site of lesion, and prognosis in 101 patients with (BP).

Materials and Methods This is a cross-sectional study conducted tertiary referral center, JSS Hospital, Mysuru. All patients consenting to participate in this study, of all ages, of either sex, both outpatients and inpatients with a diagnosis of BP during this study period were included.

Results Of the 101 patients analyzed, maximum cases (25.7\%) were in third decade of age; $55.4 \%$ were males, and both right and left sides of the face were equally involved. Maximum number of patients (50.5\%) had a history of postaural pain at presentation Topodiagnostic tests showed majority of BP cases involving geniculate or suprageniculate regions (67.3\%) in our study. $20.8 \%$ had lesion above the nerve to stapedius, and $11.9 \%$ had lesion below the nerve to stapedius. Electrodiagnostic test-electroneuromyography (ENMG)-was abnormal in 57 (67.1\%) individuals at day $4.50 .4 \%$ of patients had a House-Brackmann (HB) facial nerve grade IV at presentation. $90 \%$ of the BP group in our study recovered normal to near-normal facial nerve function by the end of 28 days' time. Conclusion Each case of BP should be evaluated with thorough clinical examination, topodiagnostic tests, and electrodiagnostic tests. Appropriate management will help in almost full recovery of disease.
\end{abstract}

\section{Introduction}

Being a mixed nerve, facial nerve supplies motor fibers to muscles of facial expression and stapedius muscle; parasympathetic secretomotor fibers to lacrimal gland, submandibular, and sublingual gland; and taste fibers to anterior two-thirds of the tongue. Its paralysis can lead to mechanical impairment as well as emotional and social implications. The most common cause of lower motor nerve (LMN) palsy is Bell's palsy (BP) $(70 \%)^{1}$ that is defined as an acute-onset, idiopathic, unilateral, LMN facial paresis (partial diminished movement), or paralysis (complete loss of movement), with no identifiable etiologies, such as infection, neoplasm, or trauma. ${ }^{2}$ Onset is typically abrupt and gradually worsens over 2 to 3 days. A preceding viral illness may be noted in up to $60 \%$ of these patients. ${ }^{3}$ Acute inflammation and edema of the facial nerve leads to entrapment of the nerve in the bony canal (as it runs through bony canal in intratemporal course), thus resulting in compression ischemia. ${ }^{4}$ Patients with acute Bell's palsy BP show facial nerve enhancement on magnetic resonance imaging (MRI), thus supporting an inflammatory etiology. ${ }^{5}$ Patients complain of deviation of the angle of mouth, incomplete eye closure, hyperacusis, taste disturbances, and decreased lacrimation. Majority of 
cases are self-limiting, nonprogressive, and spontaneously remitting, although few patients may present with residual neurologic dysfunction. ${ }^{6}$ Topodiagnostic tests are required to know the site of lesion. Electrodiagnostic tests offer the potential advantage of an objective registration of electrically evoked responses. The paralysis graduation is important for the grading severity and for clinical follow-up. Various methods of grading have been proposed along the years, and the scores of House-Brackmann (HB) are widely used. ${ }^{7}$ This study focuses on the demographic characteristics of BP, site of lesion, severity of paralysis, and recovery of patients presenting to tertiary care referral hospital.There has been a need for a study of this kind to investigate the recovery of patients to aid in better management of these individuals including the role of surgical exploration of the facial nerve in the selected few.

\section{Materials and Methods}

\section{Type of Study}

This was a cross-sectional study.

\section{Duration of the Study}

Data were collected for 2 years (October 2015 to September 2017), followed by analysis and write-up.

\section{Inclusion Criteria}

All patients consenting to participate in this study, of all ages, of either sex, both outpatients and inpatients with a diagnosis of BP during this study period and presenting to Otorhinolaryngology, Neurology, and General Medicine departments in our hospital were included.

\section{Exclusion Criteria}

Patients with upper motor neuron (UMN) facial palsy (multiple sclerosis, stroke, tumor), other etiologies of LMN facial palsy (temporal bone trauma, acute suppurative otitis media [ASOM], chronic suppurative otitis media [CSOM], Ramsay Hunt syndrome, parotid abscess, parotid malignancy, iatrogenic causes, human immunodeficiency syndrome [HIV] infection, Lyme disease, sarcoidosis, Hansen's disease, facial nerve tumor) and patients with other cranial nerve palsies were excluded clinically.

\section{Methodology}

Patient information was collected after obtaining informed consent. Patients were diagnosed to have LMN facial palsy if muscles of facial expression were involved on the same side of nerve involvement. Idiopathic cases of LMN palsy were included as BP for this study after excluding other etiologies for LMN palsy.

Careful history regarding the age; sex; onset; duration and progression of paralysis; and associated symptoms such as ear pain, dry eye, and altered taste was taken in a semi structured proforma. Patients with onset of paralysis within 72 hours were included for this study.

Complete ENT (ear-nose-throat) and head and neck evaluation including neurological examination was done.
Topodiagnostic tests such as Schirmer's test, stapedial reflex, and taste sensation testing were performed. Electrodiagnostic tests, nerve excitability test, and maximum stimulation test rely on subjective observations and can be observer biased, hence not used. In contrast, electroneuromyography (ENMG), an objective test, was used at day 4 of presentation. It records supramaximum stimulation-evoked compound action potentials (CAPs) from muscles, and the amplitude of response on the paralyzed side can be expressed as a percentage of that on the healthy side. Loss of more than $50 \%$ of amplitude compared with normal side was taken as abnormal. HB facial nerve grading system was used to assess the severity of lesion at the day of presentation. ${ }^{8}$

Laboratory and imaging studies were not routinely used in the diagnosis of BP as they are only recommended in patients with recurrence or if the condition does not resolve after more than 3 weeks of therapy. ${ }^{9}$

Patients were put on oral steroids. The prednisolone dose used was $60 \mathrm{mg} /$ day for 5 days then tapered over next 10 days. Acyclovir $400 \mathrm{mg}$ five times per day for 7 days or valacyclovir $1 \mathrm{~g}$ three times per day for 7 days was added. Patients were followed up on days 14 and 28 of presentation, and HB grading was reassessed to observe and document objective improvement.

\section{Results}

A total of 101 patients with BP were enrolled for this study. $25.7 \%$ of patients were in the age group of 31 to 40 years, followed by $20.8 \%$ in 21 to 30 years. BP was equally distributed at $13.9 \%$ in both $<20$ and 41 to 50 years and least $(7.9 \%)$ in $>61$ years of age group (- Table 1). Males constituted 55.4\% (56) and females $44.6 \%$ (45) in the study ( - Fig. 1). Right-sided BP was seen in 50 patients and left side in 51 (-Table 2). 50.5\% of patients had a history of postaural pain at presentation, and $8.9 \%$ had history of dry eye. Altered taste was reported in $12.8 \%$ individuals. Hyperlacrimation and aural fullness, each was reported in $4.9 \%$ and $8.9 \%$ individuals, respectively. Hyperacusis just prior to the onset of facial weakness was forthcoming in 14 (13.8\%) individuals (-Table 3). 68 (67.3\%) of individuals had dry eye (Schirmer's strip wetting $<15 \mathrm{~mm}$ ) on the affected side indicating geniculate or suprageniculate involvement, and 33\% had normal Schirmer's test on affected aide (-Table 4). Stapedial reflex was abnormal on the affected side in $89(88.1 \%)$ individuals. Thus $89-68=21(20.8 \%)$ patients had lesion above the nerve to stapedius. Also, this test was abnormal in $31.7 \%$ of individuals on the unaffected

Table 1 Age distribution among patients with Bell's palsy

\begin{tabular}{|l|l|l|}
\hline Age category $(y)$ & Count & Percentage \\
\hline$<20$ & 14 & 13.90 \\
\hline $21-30$ & 21 & 20.80 \\
\hline $31-40$ & 26 & 25.70 \\
\hline $41-50$ & 14 & 13.90 \\
\hline $51-60$ & 18 & 17.80 \\
\hline$>61$ & 8 & 7.90 \\
\hline
\end{tabular}




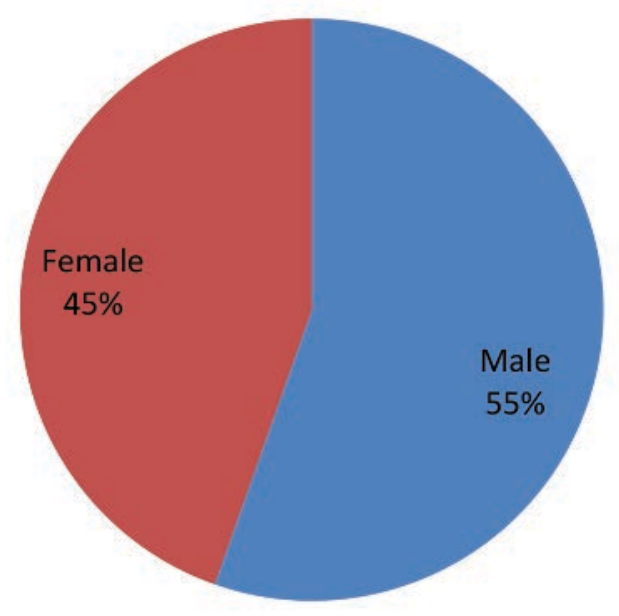

Fig. 1 Pie chart showing the sex distribution of study population.

Table 2 Side affected in Bell's palsy

\begin{tabular}{|l|l|l|}
\hline $\begin{array}{l}\text { Side of face } \\
\text { affected }\end{array}$ & Count & Percentage \\
\hline Right & 50 & 49.50 \\
\hline Left & 51 & 50.50 \\
\hline total & 101 & \\
\hline
\end{tabular}

Table 3 Distribution of various symptoms among patients

\begin{tabular}{|l|l|l|}
\hline $\begin{array}{l}\text { Associated symptoms at } \\
\text { presentation }\end{array}$ & Count & Percentage \\
\hline Postaural pain & 51 & 50.50 \\
\hline Dry eye & 9 & 8.90 \\
\hline Altered taste & 13 & 12.80 \\
\hline Hyper lacrimation & 5 & 4.90 \\
\hline Aural fullness & 9 & 8.90 \\
\hline Hyperacusis & 14 & 13.80 \\
\hline
\end{tabular}

Table 4 Percentage of dry eye on Schirmer's test in Bell's palsy

\begin{tabular}{|l|l|l|}
\hline Taste sensation & Count & Column N (\%) \\
\hline Normal & 33 & 32.7 \\
\hline Dry eye & 68 & 67.3 \\
\hline Total & 101 & \\
\hline
\end{tabular}

side (-Fig. 2). The remaining 12 (11.9\%) had lesion below the nerve to stapedius ( $\boldsymbol{-}$ Fig. 3). Taste sensation was absent in $76(75.3 \%)$ individuals on the affected side (- Table 5). Of the 101 patients, 85 underwent ENMG. It was abnormal in $57(67.1 \%)$ individuals at day 4 (-Table 6). In our study, at presentation, $50.4 \%$ of patients had HB grade IV, followed by $30.7 \%$ with grade III and $10.9 \%$ grade II facial palsy. Grade V and grade VI palsies were seen in 6 and $2 \%$ of the individuals, respectively. Of these, 11 (10.9\%) individuals at day 14 and 50 (49.5\%) at day 28 achieved normal facial nerve function (HB grade I). Fifty-seven (56.4\%) individuals at day 14 and 42 (41.5\%) at day 28 achieved HB grade II, 24 (23.7\%) at day 14 and 7 (7\%) at day 28 had HB grade III facial nerve function, $1 \%$ individuals at day14 and day 28 had HB grade V residual facial palsy, and none of the individuals had $\mathrm{HB}$ grade VI facial palsy at the end of 28 days (-Fig. 4 ).

\section{Discussion}

LMN facial palsy is a very common clinical occurrence with an estimated incidence of 20 to 30 per 100,000 population. ${ }^{10}$ $\mathrm{BP}$ is the most common cause for LMN palsy.

A total of 101 patients of BP were enrolled in this study. Maximum numbers (25.7\%) of patients were from 31 to 40 years of age. Holland and Weiner ${ }^{11}$ reported the highest incidence in the 15- to 45-year age group, whereas Prescott ${ }^{12}$ reported peak incidence in second to fourth decades. The male-to-female ratio for BP is approximately equal as given by Holland and Weiner. ${ }^{11}$ However, in this study, males constituted 55.4\% (56) and females constituted 44.6\% (45) patients. As observed in several studies, even in our study, the left and

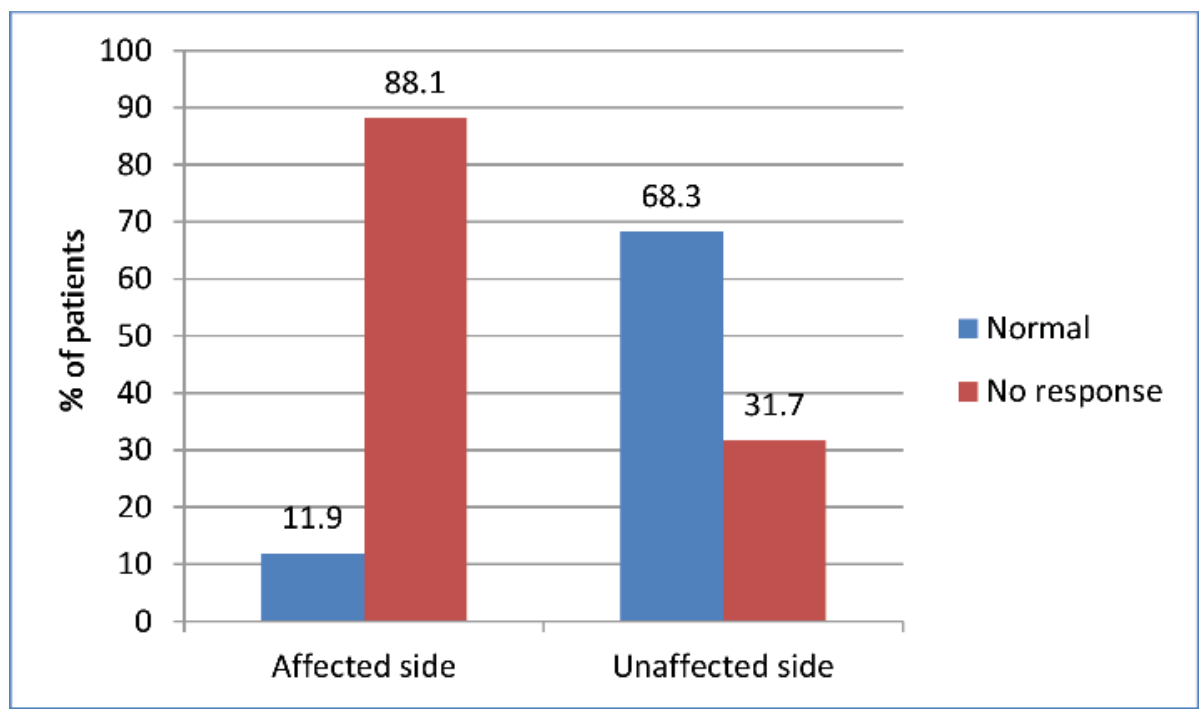

Fig. 2 Bar graph showing comparison of stapedial reflex on affected and unaffected sides. 


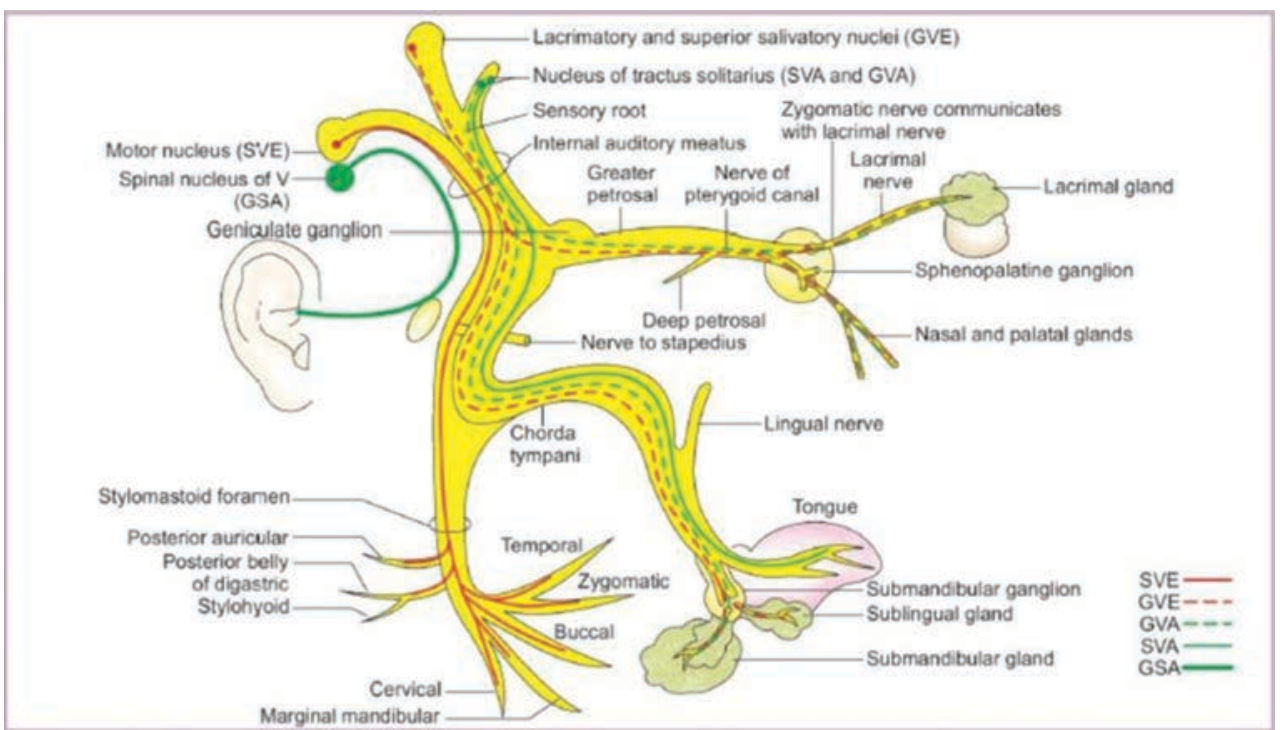

Fig. 3 Schematic diagram showing level of lesion in Bell's palsy group. Reproduced with permission from CBS Publishers and Distributors Pvt. Ltd. (BD Chaurasia, Human Anatomy. 8th ed. 2009:80)

Table 5 Individuals with normal and absent taste sensation

\begin{tabular}{|l|l|l|}
\hline Taste sensation & Count & Column N (\%) \\
\hline Absent & 76 & 75.30 \\
\hline Present & 35 & 34.70 \\
\hline
\end{tabular}

Table 6 ENMG results in the study population

\begin{tabular}{|l|l|}
\hline ENMG & Day 4 \\
\hline Normal & $28(32.9 \%)$ \\
\hline Abnormal & $57(67.1 \%)$ \\
\hline Total patients undergoing test & 85 \\
\hline Test not done & 16 \\
\hline
\end{tabular}

Abbreviation: ENMG, electroneuromyography.

right sides of the face were equally involved. ${ }^{13}$ In our study, maximum patients (50.5\%) had postaural pain as an associated symptom at presentation, which was similar to the study by De Seta et al. ${ }^{13}$ Topodiagnostic tests showed majority of $\mathrm{BP}$ cases involving the geniculate or suprageniculate region (67.3\%) in our study. This is in accordance with the reports by Renou and Psaume. ${ }^{14}$ The authors noted the existence of a geniculate or suprageniculate lesion in $62 \%$ of cases. Stapedial reflex was abnormal on the unaffected side in some patients (31.7\%) possibly due to subclinical involvement of the unaffected side in BP. Taste sensation was found to be abnormal in 76 individuals, suggesting involvement above the chorda tympani branch. However, $(68+21) 89$ patients should already have loss of taste. This paradox could possibly be explained by incomplete and patchy involvement of the facial nerve in BP. Unfortunately, the accuracy of localization with topodiagnostic testing has been disappointing. ${ }^{15}$ This is not a surprising finding in BP in which the lesion is a diffuse demyelination throughout the nerve. In contrast to traumatic injuries, BP is typically a mixed and partial lesion with varying degrees of conduction block and degenerative changes within different fibers and fascicles of the nerve trunk; therefore, topodiagnostic tests are not expected to provide precise information about the level of the lesion. In our study, we found disparate results of BP with respect to topodiagnostic tests.

Electrodiagnostic tests-ENMG studies-of the facial nerve can yield useful information on the viability of the affected nerve, thus aiding in the decision-making process regarding treatment and/or surgical management of disease. ${ }^{16}$ These electrodiagnostic studies yield information on the amount of evoked action potentials in affected muscles. Using these data, clinicians can estimate the amount of axonal loss. Patients exhibiting more than $90 \%$ axonal degeneration should be considered for surgical decompression, whereas axonal degeneration of less than $90 \%$ has a favorable prognosis. ${ }^{17}$ None of the patients in our study had degeneration more than $90 \%$. These tests have a shortcoming: stimulation and recording are performed distal to the lesion. Therefore, it is prudent to wait until nerve degeneration has reached the site of stimulation; it usually takes 4 to 5 days before electrodiagnostic tests become abnormal. This may be the reason for the test not being abnormal in $100 \%$, despite facial palsy being appreciable clinically. In the study by Gantz et al, ${ }^{18}$ all patients who had less than $90 \%$ neural degeneration within 14 days of total facial paralysis recovered normal to near-normal facial function, $89 \%$ with HB grades I and $11 \%$ with HB grade II. In our study, 67\% had near-normal facial function (HB grades I and II) by day 14 and did not agree for repeat ENMG.

Combination therapy was started for treatment as two recent placebo-controlled trials established full recovery in a higher percentage of patients after combined therapy with an antiviral drug and prednisolone than with prednisolone alone (100 vs. $91 \%$ and 95 vs. $90 \%$ ). ${ }^{19,20}$

In our study, at presentation, maximum percentage (50.4\%) patients had an HB grade IV of the BP. In the study by De Seta et $a l,{ }^{13}$ maximum number of patients (35\%) had grade III palsy. $91 \%$ of the BP group in our study recovered normal to near-normal facial nerve function (HB grades I and 


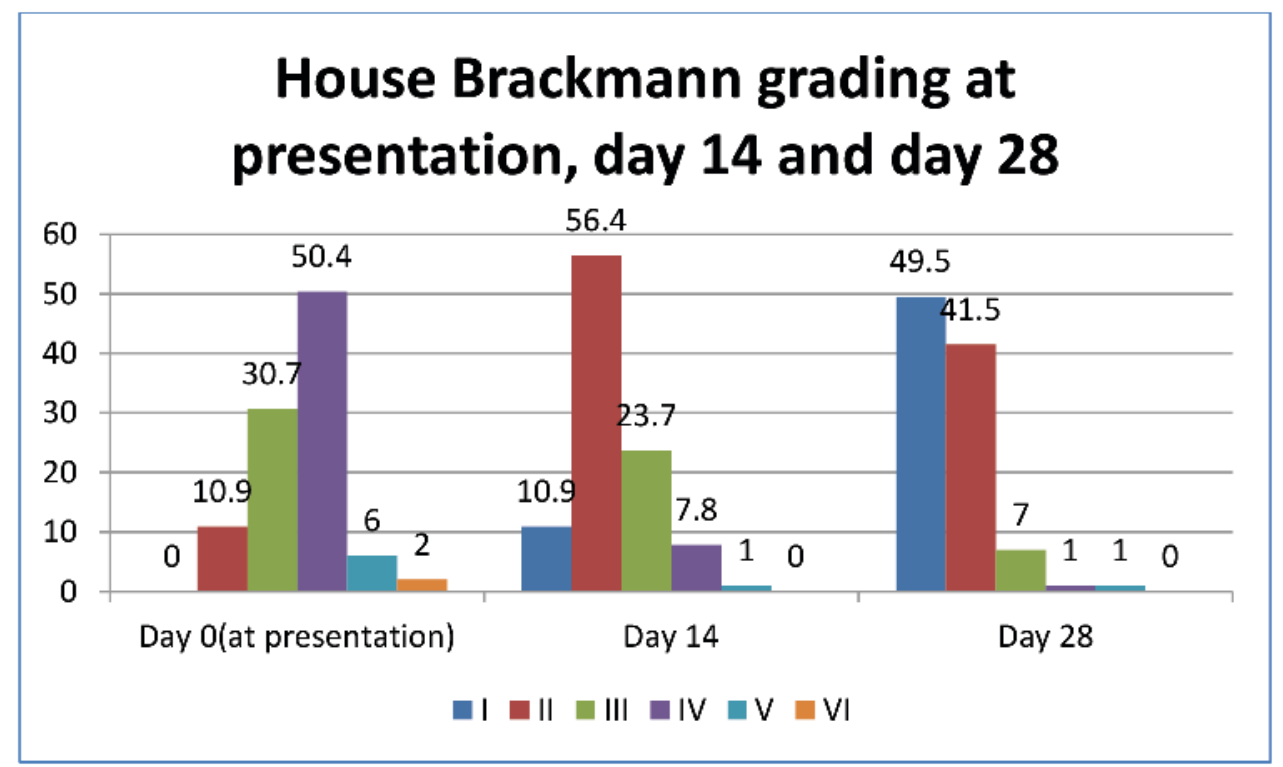

Fig. 4 Bar graph showing the recovery of patients with Bell's palsy on House-Brackmann grading at presentation, day 14, and day 28.

II) by the end of 28 days' time. Similar findings by Yeo et $\mathrm{al}^{7}$ in 2007 suggested that $96.3 \%$ of patients with BP recovered spontaneously. Hence, the role of facial nerve decompression surgery is limited to the extreme few.

\section{Conclusion}

Bell's palsy, also known as idiopathic facial palsy, is a common LMN disorder with an obscure etiopathogenesis. Diagnosis is usually made by exclusion, and treatment of this psychologically distressing condition remains controversial till date, but it has very good prognosis.

\section{Funding}

None.

\section{Conflict of Interest \\ None declared.}

\section{References}

1 Adour KK, Byl FM, Hilsinger RL Jr, Kahn ZM, Sheldon MI. The true nature of Bell's palsy: analysis of 1,000 consecutive patients. Laryngoscope 1978;88(5):787-801

2 Miller NR, Newman NJ, eds. Walsh \& Hoyt's Clinical Neuro- Ophthalmology: The Essentials. 5th ed. Baltimore, MD: Williams \& Wilkins; 1999

3 Benecke JE Jr. Facial paralysis. Otolaryngol Clin North Am 2002;35(2):357-365

4 Zandian A, Osiro S, Hudson R, et al. The neurologist's dilemma: a comprehensive clinical review of Bell's palsy, with emphasis on current management trends. Med Sci Monit 2014;20:83-90

5 Schwaber MK, Larson TC III, Zealear DL, Creasy J. Gadolinium-enhanced magnetic resonance imaging in Bell's palsy. Laryngoscope 1990;100(12):1264-1269

6 Amit R. Familial juvenile onset of Bell's palsy. Eur J Pediatr 1987;146(6):608-609
7 Yeo SW, Lee DH, Jun BC, Chang KH, Park YS. Analysis of prognostic factors in Bell's palsy and Ramsay Hunt syndrome. Auris Nasus Larynx 2007;34(2):159-164

8 House JW, Brackmann DE. Facial nerve grading system. Otolaryngol Head Neck Surg 1985;93(2):146-147

9 Bodénez C, Bernat I, Willer JC, Barré P, Lamas G, Tankéré F. Facial nerve decompression for idiopathic Bell's palsy: report of 13 cases and literature review. J Laryngol Otol 2010;124(3):272-278

10 Pereira CU, Santos EA, Monteiro J, et al. Peripheral facial palsy: anatomy and physiology. An update. Int J Neurosurg 2005;2(2):xx

11 Holland NJ, Weiner GM. Recent developments in Bell's palsy. BMJ 2004;329(7465):553-557

12 Prescott CA. Idiopathic facial nerve palsy (the effect of treatment with steroids). J Laryngol Otol 1988;102(5):403-407

13 De Seta D, Mancini P, Minni A, et al. Bell's palsy: symptoms preceding and accompanying the facial paresis. ScientificWorldJournal 2014;2014:801971

14 Renou G, Psaume T. [Topographical diagnosis of facial paralysis (Bell's palsy) (author's transl)]. Ann Otolaryngol Chir Cervicofac 1978;95(12):769-775

15 Gates GA. Nerve excitability testing: technical pitfalls and threshold norms using absolute values. Laryngoscope 1993;103(4 Pt 1):379-385

16 Valls-Solé J. Electrodiagnostic studies of the facial nerve in peripheral facial palsy and hemifacial spasm. Muscle Nerve 2007;36(1):14-20

17 Schularick NM, Mowry SE, Soken H, Hansen MR. Is electroneurography beneficial in the management of Bell's palsy? Laryngoscope 2013;123(5):1066-1067

18 Gantz BJ, Rubinstein JT, Gidley P, Woodworth GG. Surgical management of Bell's palsy. Laryngoscope 1999;109(8):1177-1188

19 Hato N, Yamada H, Kohno H, et al. Valacyclovir and prednisolone treatment for Bell's palsy: a multicenter, randomized, placebo-controlled study. Otol Neurotol 2007;28(3):408-413

20 Hato N, Matsumoto S, Kisaki H, et al. Efficacy of early treatment of Bell's palsy with oral acyclovir and prednisolone. Otol Neurotol 2003;24(6):948-951 